\title{
26295 - LUMBAR DURAL SAC WIDTH BY ULTRASOUND AND SENSORY LEVELS UNDER SPINAL
}

\section{Cristian Arzola MD, Cristian Arzola, MD; Mrinalini Balki, MD; Jose Carvalho, Mount Sinai Hospital And University Of Toronto, Toronto, ONTARIO, Canada}

INTRODUCTION: The lumbosacral CSF volume, as assessed by MRI(1,2), has been reported to be one of the key factors influencing intrathecal spread of local anesthetics. Ultrasound imaging of the lumbar spine, although not able to determine CFS volume, allows us to assess the width of the lumbar dural sac (DSW)(3). One could hypothesize that DSW is an estimation of CSF volume. The purpose of this study was to investigate whether DSW correlates with sensory levels of spinal anesthesia for elective Cesarean sections (CS).

METHODS: After REB approval and written informed consent, 22 patients scheduled for elective CS under spinal anesthesia were enrolled. The dural sac width (DSW=distance between the inner surfaces of the dural sac) was measured by ultrasound imaging performed with the patient in the sitting position, at the L3/4 interspace, using the transverse approach, with a portable Titan Ultrasound System equipped with a 5.0-MHz curved array probe (Sonosite Canada Inc.). Spinal anesthesia [1.6 ml hyperbaric $0.75 \%$ bupivacaine + fentanyl $0.2 \mathrm{ml}(10 \mathrm{mcg})+$ morphine $0.2 \mathrm{ml}(100 \mathrm{mcg})]$ was administered at L3-L4, with the patients in the sitting position, using a $27 \mathrm{G}$ Whitacre needle, with the needle aperture directed cephalad. The intrathecal solution was injected over 30 seconds. After the intrathecal injection, the patients assumed the supine position with a left lateral tilt. Sensory block levels were assessed bilaterally, in the midclavicular line, by ice and pinprick (25-gauge needle). Assessments were done every 5 minutes until peak sensory levels (PSL) were achieved. Spearman's rank correlation was used to correlate PSL and time to develop PSL with DSW. Multiple linear regression analysis was used to estimate the influence of patients' demographics on sensory block levels.

RESULTS: Patient demographics, DSW values and sensory block levels are shown in the table. There were no significant correlations between PSL and DSW. DSW appeared to be an independent variable evaluated by Spearman's rank correlation. Multiple regression analysis showed no influence of patients' demographics on PSL.

DISCUSSION: The lumbar dural sac width, as determined by ultrasound, is not a predictor of spinal anesthesia spread. Further studies are necessary to understand how ultrasound findings can translate specific compartments in the spine and therefore be useful predictors of spinal spread of local anesthetics.

REFERENCES: 1) Anesthesiology1998;89:24-29; 2)Anesthesiology 2004;100:106-14;

3)Can J Anaesth 2003;50:R1-R8 


\begin{tabular}{|l||l|}
\hline Patient demographics & $n=22$ \\
\hline Age $(\mathrm{yr})$ & $35.4 \pm 4.4$ \\
\hline Height $(\mathrm{cm})$ & $162.9 \pm 6.5$ \\
\hline Weight $(\mathrm{kg})$ & $82.8 \pm 14.6$ \\
\hline BMI $\left(\mathrm{kg} / \mathrm{m}^{2}\right)$ & $31.2 \pm 5.5$ \\
\hline Neonatal weight $(\mathrm{g})$ & $3445 \pm 497$ \\
\hline Dural sac width $(\mathrm{cm})$ & $0.79 \pm 0.13(0.59-1.17)$ \\
\hline Sensory block & \\
\hline Peak level to ice & $\mathrm{T} 3(\mathrm{~T} 1-\mathrm{T} 4)$ \\
\hline Peak level to pinprick & $\mathrm{T} 3(\mathrm{~T} 1-\mathrm{T} 5)$ \\
\hline Time to peak level to ice (min) & $12.5(5-20)$ \\
\hline Time to peak level to pinprick (min) & $15(5-20)$ \\
\hline \hline & \\
\hline \hline & \\
\hline values are mean \pm SD or median, with ranges in parenthesis \\
\hline
\end{tabular}

